K. Yamato

Nagoya Math. J.

Vol. 123 (1991), 77-90

\title{
A CHARACTERIZATION OF LOCALLY HOMOGENEOUS RIEMANN MANIFOLDS OF DIMENSION 3
}

\author{
KAZUO YAMATO
}

\section{Introduction}

It is classical to characterize locally homogeneous Riemann manifolds by infinitesimal conditions. For example, [Si] asserts that the localhomogeneity is equivalent to the existence of linear isometries between tangent spaces which preserve the curvatures and their covariant derivatives up to certain orders. It is also known that the local homogeneity is equivalent to the existence of a certain tensor field of type $(1,2)$ (for this and a further study, see [TV]).

In connection with his characterization theorem, Singer raised the following questions:

(Q1) What are the Riemann manifolds which are completely determined by their curvatures only?

(Q2) Do there exist curvature homogeneous spaces which are not locally homogeneous?

The purpose of the present paper is to give, in the 3-dimensional case, an explicit characterization (i.e. in terms of Riemannian invariants) of locally homogeneous Riemann manifolds, and to give some answers to the questions of Singer.

Our characterization is as follows: Let $M$ be a connected, compact Riemann manifold of dimension 3 and $S$ the Ricci tensor. Assume that the eigenvalues $\rho_{1}, \rho_{2}, \rho_{3}$ of $S$ are constant on $M$ (in other words we assume that $M$ is curvature homogeneous).

Theorem A. Suppose that $\rho_{1}, \rho_{2}, \rho_{3}$ are distinct. Then $M$ is locally homogeneous if and only if the 1-form $S \cdot \nabla S=\sum_{a, b} S^{a b} S_{i a ; b}$ vanishes. If that is the case, then $\rho_{1}, \rho_{2}, \rho_{3}$ give complete isometry invariants for the universal covering manifold of $M$.

Received July 10, 1990. 
Theorem B. Suppose that two of $\rho_{1}, \rho_{2}, \rho_{3}$ coincide. Then $M$ is locally homogeneous if and only if the function $\|\nabla S\|^{2}=\sum_{a, b, c} S^{a b ; c} S_{a b ; c}$ is constant. In that case, if $\rho_{i}=\rho_{j}=0 \neq \rho_{k}$ for some distinct $i, j, k$, then $\rho_{k},\|\nabla S\|$ give complete isometry invariants for the universal covering manifold, and otherwise $\rho_{1}, \rho_{2}, \rho_{3}$ give those.

From the proof of Theorem B we obtain a sufficient condition for the local-homogeneity of $M$ :

Proposition 5.1. Suppose that $\rho_{1}=\rho_{2}$. If $\rho_{1} \geq 0$ or $\rho_{3} \leq 0$, then $M$ is locally homogeneous.

This proposition (with Theorem B) gives an answer in the 3-dimensional case to question (Q1). We also note that the assumption of compactness of $M$ is essential in Proposition 5.1. Indeed, on $\mathbf{R}^{3}$, there exist nonhomogeneous, complete metrics with $\rho_{1}=\rho_{2}=-1, \rho_{3}=0$ ([Se], [T], [KTV]).

We also give examples of non-homogeneous, complete metrics on $\mathbf{R}^{3}$ which have distinct, constant Ricci eivenvalues $(\S 6)$. These give counterexamples in the non-degenerate case $\rho_{i} \neq \rho_{j}(i \neq j)$ to question (Q2).

To obtain our result we proceed as follows. The proof of Theorem A is rather straightforward, because the assumption of the distinctness of the Ricci eigenvalues ensures the uniqueness of the Ricci eigenvector fields, and hence the isometry between neighborhoods of two points, if any, is also uniquely determined. On the other hand, if the Ricci eigenvalues are not distinct, say $\rho_{1}=\rho_{2} \neq \rho_{3}$, there are two possibilities: the one where the required isometry is uniquely determined and the other where the isometry is not uniquely determined, in other words, the isotropy group is nontrivial. In order to distinguish these cases, we introduce an isometry invariant function $\delta$ so that for a point $p$, the nontriviality of the isotropy group at $p$ implies $\delta(p)=0$, and $\delta$ being constant yields the local-homogeneity of $M$. The function $\delta$ is expressed by $S$ and $\nabla S$, and hence the condition that $\|\nabla S\|^{2}$ be constant as in Theorem B means that $\delta$ is constant. The main task in the proof of Proposition 5.1 is the proof of $\delta$ being constant, which is done by applying the geometric version of E. Hopf theorem to the function $\delta$ and some elliptic operator defined by means of Ricci eigenvector fields.

Throughout this paper, all manifolds and functions are assumed to be of class $C^{\infty}$. 


\section{§1. Preliminary formulas}

Let $U$ be a 3-dimensional open ball with Riemann metric $\langle$,$\rangle . Assume$ that the eigenvalues $\rho_{1}, \rho_{2}, \rho_{3}$ of the Ricci tensor $S$ (viewed as a tensor of type $(1,1))$ are constant on $U$. Let $\left(X_{1}, X_{2}, X_{3}\right)$ be the orthonormal frame field such that each $X_{i}$ is the eigenvector field belonging to $\rho_{i}$ (although such a frame is not unique in the degenerate cases $\rho_{i}=\rho_{j}$ ). Introduce the functions $d_{i j}^{k}=\left\langle\nabla_{X_{i}} X_{j}, X_{k}\right\rangle$ on $U$. Then $d_{i j}^{k}=-d_{i k}^{j}$, $\operatorname{div} X_{i}=-\sum_{a} d_{a a}^{i}$, and the following formulas are directly verified.

Lemma 1.1. For each $i, j, l=1,2,3$, we have

(i,i) $\quad \rho_{i}=-X_{i}\left(\operatorname{div} X_{i}\right)+\sum_{a} X_{a}\left(d_{i i}^{a}\right)+\sum_{a} d_{i i}^{a} \operatorname{div} X_{a}-\sum_{a, b} d_{b i}^{a} d_{a i}^{b}$,

$(j, l) \quad 0=-X_{j}\left(\operatorname{div} X_{l}\right)+\sum_{a} X_{a}\left(d_{j l}^{a}\right)+\sum_{a} d_{j l}^{a} \operatorname{div} X_{a}-\sum_{a, b} d_{b j}^{a} d_{a l}^{b}, \quad j \neq l$,

and as Bianchi's 2nd identity,

$$
\sum_{a}\left(\rho_{i}-\rho_{a}\right) d_{a a}^{i}=0 .
$$

In particular, if $\rho_{1}=\rho_{2} \neq \rho_{3}$, then

$$
d_{33}^{1}=d_{33}^{2}=0, \quad d_{11}^{3}+d_{22}^{3}=0, \quad \rho_{3}=2\left(d_{12}^{3} d_{23}^{1}-\left(d_{11}^{3}\right)^{2}\right) .
$$

Hence, in the case $\rho_{1}=\rho_{2} \neq \rho_{3}$, every integral curve for $X_{3}$ is a geodesic, and the divergence $\operatorname{div} X_{3}$ of $X_{3}$ vanishes.

Lemma 1.2. Assume that $\rho_{1}=\rho_{2} \neq \rho_{3}$. Define $\delta: U \rightarrow \mathbf{R}$ by the formula

$$
\delta(x)=\operatorname{det}\left(\nabla X_{3} \mid P+\left(\nabla X_{3} \mid P\right)^{*}\right), \quad x \in U,
$$

where $\nabla X_{3} \mid P$ denotes the restriction to $P=\left(X_{3}\right)_{\frac{\perp}{x}}$ of the linear mapping $\nabla X_{3}: T_{x}(U) \rightarrow T_{x}(U)$, and $\left(\nabla X_{3} \mid P\right)^{*}$ its adjoint. Then $\delta$ is independent of the choice (i.e. the orientation) of $X_{3}$, and satisfies $\delta \leq 0$. Furthermore we have $X_{3}(\delta)=0$.

Proof. Note that

$$
\delta=-4\left(d_{11}^{3}\right)^{2}-\left(d_{12}^{3}-d_{23}^{1}\right)^{2}=2 \rho_{3}-\left(d_{12}^{3}+d_{23}^{1}\right)^{2} .
$$

Then the former parts of our lemma are obvious. By $(1,2),(2,1)$ of Lemma 1.1 we have $X_{3}\left(d_{12}^{3}+d_{23}^{1}\right)=0$, which implies the latter part. This expression for $\delta$ also yields

Lemma 1.3. Let the assumption and the notation be as in Lemma 1.2. 
If $\delta \equiv 0$, then

$$
d_{11}^{3}=d_{22}^{3}=0, \quad d_{12}^{3}=d_{23}^{1}, \quad \rho_{3}=2\left(d_{12}^{3}\right)^{2} .
$$

In the case where $\delta<0$ on $U$, the following lemma gives us a "canonical" frame $\left(X_{1}, X_{2}, X_{3}\right)$ for the Ricci tensor $S$.

Lemma 1.4. Let the assumption and the notation be as in Lemma 1.2. Suppose that $\delta(x)<0$ for any $x \in U$. Then we can take $X_{1}, X_{2}$ so that $d_{11}^{3}=d_{22}^{3}=d_{31}^{2}=0, d_{12}^{3}>d_{23}^{1}$ at every point of $U$, and so that $d_{12}^{3}+d_{23}^{1}>0$ at some point of $U$ or $d_{12}^{3}+d_{23}^{1}=0$ at every point of $U$.

Proof. Let $\left(Y_{1}, Y_{2}\right)$ be the orthonormal frame field diagonalizing the symmetric operator $\nabla X_{3} \mid P+\left(\nabla X_{3} \mid P\right)^{*}$. (Since $\left(Y_{1}, Y_{2}\right)$ is uniquely determined up to permutations, they are globally defined on $U$.) Rotate $\left(Y_{1}, Y_{2}\right)$ by $\pi / 4$ to get $\left(X_{1}, X_{2}\right)$. Then $\left(X_{1}, X_{2}, X_{3}\right)$ satisfies $d_{11}^{3}=d_{22}^{3}=0$. By formulas $(1,1),(2,2)$ in Lemma 1.1 we have also $d_{31}^{2}=0$. Since $d_{12}^{3}-d_{23}^{1} \neq 0$ on $U$, by reversing the orientation of $X_{1}$ if necessary, we may assume $d_{12}^{3}-d_{23}^{1}$ $>0$ on $U$. Moreover, if $d_{12}^{3}+d_{23}^{1}$ is negative at some point of $U$, then we exchange $X_{1}, X_{2}$. Then the new vector fields satisfy the desired properties.

\section{§2. Local theory for the case $\rho_{1}=\rho_{2} \neq \rho_{3}$}

Let $U$ be as in $\S 1$ and we continue to consider the degenerate case $\rho_{1}=\rho_{2} \neq \rho_{3}$. Let $\delta$ be as in Lemma 1.2. The purpose of this section is to prove

Proposition 2.1. If $\delta$ is constant, then $U$ is locally homogeneous, i.e. for any two points $p, q$ of $U$ there exists an isometry, taking $p$ to $q$, of $a$ neighborhood of $p$ onto a neighborhood of $q$.

We prepare some lemmas.

Lemma 2.2. Assume that $\delta \equiv 0$. Then $\rho_{3} \geq 0$, and the eigenvector field $X_{3}$ belonging to $\rho_{3}$ is a Killing vector field. Moreover, for any point $p$ of $U$ there exist orthonormal vector fields $Y_{1}, Y_{2}$ on a neighborhood of $p$ such that

(1) $\left\langle Y_{i}, X_{3}\right\rangle=0,\left[Y_{i}, X_{3}\right]=0$ for $i=1,2$,

(2) $\left\langle\left[Y_{1}, Y_{2}\right], X_{3}\right\rangle=\sqrt{2 \rho_{3}}$, and hence

(3) $\rho_{1}+\rho_{3}=Y_{1}\left(c_{12}^{2}\right)-Y_{2}\left(c_{12}^{1}\right)-\left(c_{12}^{2}\right)^{2}-\left(c_{12}^{1}\right)^{2}$, where $c_{i j}^{k}=\left\langle\left[Y_{i}, Y_{j}\right], Y_{k}\right\rangle$. Hence, the neighborhood of $p$ is isometric to an open subset of the total 
space of a Riemann submersion over a surface $B$ with curvature $\rho_{1}+\rho_{3}$, whose fibers (integral curves for $X_{3}$ ) are geodesics, and whose integrability tensor is equal to $\sqrt{2 \rho_{3}}$ times the area form of $B$.

Proof. Lemma 1.3 implies $\rho_{3} \geq 0$. For any orthonormal vector fields $X_{1}, X_{2}$ orthogonal to $X_{3}$, by Lemmas 1.1 and 1.3 we have $d_{12}^{3}=d_{23}^{1}$ and $d_{i i}^{3}=0, d_{33}^{i}=0$ for any $i$. These show especially that $\nabla X_{3}$ is skewsymmetric, and therefore $X_{3}$ is Killing. Since the problem is local, it is easy to take orthonormal vector fields $Y_{1}, Y_{2}$ orthogonal to $X_{3}$, defined in a neighborhood of $p$, so that $\left[Y_{1}, X_{3}\right]=\left[Y_{2}, X_{3}\right]=0$. To apply Lemmas in $\S 1$ to the orthonormal frame fields $\left(Y_{1}, Y_{2}, X_{3}\right)$, we may use the same notations $d_{i j}^{k}$ for the functions $\left\langle\nabla_{Y_{i}} Y_{j}, Y_{k}\right\rangle$, where $Y_{3}=X_{3}$. Then by Lemma 1.3, we obtain $\left\langle\left[Y_{1}, Y_{2}\right], X_{3}\right\rangle^{2}=2 \rho_{3}$. By replacing $Y_{1}$ by $-Y_{1}$, if necessary, we may assume that $\left\langle\left[Y_{1}, Y_{2}\right], X_{3}\right\rangle \geq 0$, and hence (2) is satisfied. Condition (3) follows from $(1,1),(3,3)$ of Lemma 1.1 and the fact $d_{12}^{3}+d_{31}^{2}=0$. The latter part is an immediate consequence of (1), (2), (3) and the fact that for a surface $B$ with orthonormal vector fields $Y_{1}, Y_{2}$, the curvature of $B$ is given by $\left\langle R\left(Y_{1}, Y_{2}\right) Y_{2}, Y_{1}\right\rangle=Y_{1}\left(c_{12}^{2}\right)-Y_{2}\left(c_{12}^{1}\right)-\left(c_{12}^{2}\right)^{2}-\left(c_{12}^{1}\right)^{2}$, where $c_{i j}^{k}=$ $\left\langle\left[Y_{i}, Y_{j}\right], Y_{k}\right\rangle$. This completes the proof of Lemma 2.2.

Lemma 2.3. Assume that $\delta$ is negative constant. Then the vector fields $X_{1}, X_{2}, X_{3}$ taken as in Lemma 1.4 satisfy the conditions $d_{i j}^{k}=$ constant. In fact, $d_{12}^{3}$ and $d_{23}^{1}$ are the constants determined by $\rho_{3}$ and $\delta$, and other $d_{i j}^{k}$ 's vanish. Moreover we have $\rho_{1}=\rho_{2}=0$.

Proof. We know already that all the $d_{i j}^{k}$ 's except $d_{22}^{1}, d_{11}^{2}, d_{12}^{3}, d_{23}^{1}$ are identically zero. As for $d_{12}^{3}, d_{23}^{1}$, by the expression for $\delta$ in the proof of Lemma 1.2, we have $d_{12}^{3}-d_{23}^{1}=\sqrt{-\delta}, d_{12}^{3}+d_{23}^{1}=\sqrt{2 \rho_{3}-\delta}$. Hence $d_{12}^{3}, d_{23}^{1}$ are the constants determined by $\rho_{3}, \delta$. Furthermore, by formulas $(2,3)$, $(1,3)$ of Lemma 1.1 we observe that $d_{22}^{1}=0, d_{11}^{2}=0$, respectively. Hence $(1,1)$ yields $\rho_{1}=0$. Lemma 2.3 is proved.

We need two more general lemmas.

Lemma 2.4. Let $\pi$ be a submersion of an n-dimensional Riemann manifold $M$ onto an $(n-1)$-dimensional Riemann manifold $B$ with structure tensors T, A (cf. [O]). Suppose that the submersion $\pi$ is induced by a Killing vector field, that is, there exists a non-singular Killing vector field $f$ on $M$ such that $f$ is vertical at every point of $M$. Put $\nu=f /\|f\|, N=T_{\nu} \nu$. Consider the 2-form on $M$ defined by $(X, Y) \mapsto\left\langle A_{X} Y, \nu\right\rangle$ and denote it by 
the same letter $A$. (Then we can regard $N, A$ as the vector field, 2-form on $B$, respectively.) Let $\pi^{\prime}: M^{\prime} \rightarrow B^{\prime}$ be another such submersion with $T^{\prime}$, $A^{\prime}, f^{\prime}, N^{\prime}$. Let $p \in M, p^{\prime} \in M^{\prime}$. Suppose that there exists an isometry $\phi$ of $B$ onto $B^{\prime}, \phi(\pi(p))=\pi^{\prime}\left(p^{\prime}\right)$, such that $\langle X, N\rangle=\left\langle\phi_{*} X, N^{\prime}\right\rangle$ and $A_{X} Y=$ $A_{\phi_{*} X}^{\prime} \phi_{*} Y$ for any tangent vectors $X, Y$ of $B$. Then the isometry $\phi$ can be lifted to an isometry $\bar{\phi}$ of a neighborhood of $p$ onto a neighborhood of $p^{\prime}=\bar{\phi}(p)$.

Proof. Let $p \in M$, and $\gamma(t)$ a geodesic in $B$ with $\gamma(0)=\pi(p)$. Let $\left\{\gamma_{\varepsilon}(t)\right\}$ be a family of geodesics such that $\gamma_{0}(t)=\gamma(t)$, and let $J(t)$ be the Jacobi field along $\gamma$ determined by $\left\{\gamma_{\varepsilon}(t)\right\}$. Let $\bar{\gamma}(t)$ be the horizontal lift of $\gamma(t)$ with $\bar{\gamma}(0)=p$, and let $\left\{\bar{\gamma}_{\varepsilon}(t)\right\}$ be any horizontal lift of $\left\{\gamma_{\varepsilon}(t)\right\}$ such that $\bar{\gamma}_{0}(t)=\bar{\gamma}(t)$. Let $\tilde{J}(t)$ be the Jacobi field along $\bar{\gamma}$ determined by $\left\{\bar{\gamma}_{\varepsilon}(t)\right\}$. Then we have

$$
\frac{d}{d t}\langle\tilde{J}(t), \nu\rangle=-\langle\dot{\gamma}(t), N\rangle\langle\tilde{J}(t), \nu\rangle+2\left\langle A_{J(t)} \dot{\gamma}(t), \nu\right\rangle .
$$

By this formula and the usual argument using geodesics, we can verify that the lift $\bar{\phi}$ is an isometry.

LEMma 2.5. Let $X_{1}, X_{2}, \cdots, X_{n}$ be orthonormal vector fields on a connected, simply connected Riemann manifold $U$ of dimension $n$. Let $p$ be a point of $U$. Suppose that $c_{i j}^{k}=\left\langle\left[X_{i}, X_{j}\right], X_{k}\right\rangle$ is constant on $U$ for each $i, j, k$. Then there exists a connected, simply connected Lie group $G$ of dimension $n$, which is uniquely determined by constants $c_{i j}^{k}$, such that $U \subset G$ ( $U=G$ if $U$ is complete) and $p$ is the unit element of $G$, and such that $X_{i}$ are the restrictions to $U$ of the left invariant vector fields $\tilde{X}_{i}, \tilde{X}_{i}(p)$ $=X_{i}(p)$, on $G$. The metric on $G$ defined so that $\tilde{X}_{i}$ are orthonormal is left invariant.

Proof. This is a direct consequence of the Lie's fundamental theorem on local groups of transformations.

Proof of Proposition 2.1. First consider the case $\delta \equiv 0$. Then by Lemma 2.2 we see that every point of $U$ has a neighborhood which is a total space of a submersion. Applying Lemma 2.4, since $N=0$ in this case, we conclude that the neighborhood is locally homogeneous, and hence so is $U$. Next, consider the case $\delta<0$. Then by Lemma 2.3 we can apply Lemma 2.5 to identify $U$ with an open subset of a Lie group with left invariant metric. Hence $U$ is in particular locally homogeneous. 


\section{§ 3. Proof of Theorem A}

Let $M$ be a 3-dimensional connected Riemann manifold, and let $S$, $\nabla S$ be the Ricci tensor, its covariant differential. Let $S \cdot \nabla S$ be the 1 -form defined by $(S \cdot \nabla S)_{i}=\sum_{a, b} S^{a b} S_{i a ; b}$.

We have to prove a lemma.

Lemma 3.1. Assume that the Ricci eigenvalues $\rho_{1}, \rho_{2}, \rho_{3}$ are constant and distinct. Moreover, assume that the 1-form S.VS vanishes. Let $\left(X_{1}, X_{2}, X_{3}\right)$ be the orthonormal frame field on the universal covering manifold $\tilde{M}$ of $M$ such that each $X_{i}$ is the eigenvector field belonging to $\rho_{i}$. Then the bracket products $\left[X_{i}, X_{j}\right]$ are expressed as linear combinations of $X_{1}, X_{2}, X_{3}$, whose coefficients are constants determined by $\rho_{1}, \rho_{2}, \rho_{3}$.

Proof. Since $(\nabla S)\left(X_{i}, X_{j} ; X_{k}\right)=\left(p_{i}-p_{j}\right) d_{k i}^{j}$, we note that the 1 -form $S \cdot \nabla S$ satisfies

$$
(S \cdot \nabla S)\left(X_{i}\right)=\sum_{a} \rho_{a}\left(\rho_{a}-\rho_{i}\right) d_{a a}^{i},
$$

where $d_{i j}^{k}=\left\langle\nabla_{X_{i}} X_{j}, X_{k}\right\rangle$. Then by the assumption $S \cdot \nabla S=0$ and Bianchi 2nd identity, we have $d_{j j}^{i}=0$ for each $i, j$. Hence, from formula $(i, i)$ of Lemma 1.1 we get $\rho_{i}=2 d_{j k}^{i} d_{k i}^{j}$ for any even permutation $i, j, k$ of $1,2,3$. Using the distinctness of $\rho_{i}$ we conclude that $d_{12}^{3}, d_{23}^{1}, d_{31}^{2}$ are non-zero constants, and can be expressed as functions of $\rho_{1}, \rho_{2}, \rho_{3}$. Therefore the coefficients $d_{i j}^{k}-d_{j i}^{k}$ of $\left[X_{i}, X_{j}\right]$ with respect to $X_{k}$ are the constants determined by $\rho_{1}, \rho_{2}, \rho_{3}$.

Proof of Theorem A. Suppose that $S \cdot \nabla S=0$. Then by Lemmas 2.5 and 3.1 , we can regard the universal covering manifold $\tilde{M}$ as a Lie group with left invariant metric. Hence $M$ is itself locally homogeneous. The latter part of Theorem A also follows from Lemmas 2.5, 3.1. In order to prove the necessity of the condition $S \cdot \nabla S=0$, suppose that $M$ is locally homogeneous. By considering a suitable finite covering manifold, we may assume that the Ricci eigenvector fields $X_{1}, X_{2}, X_{3}$ are defined globally on $M$. Then clearly $\operatorname{div} X_{i}$ are constant. By the compactness of $M$ we get $\operatorname{div} X_{i}=0$. These and Bianchi 2nd identity yield $d_{j j}^{i}=0$ for each $i, j$, and hence $S \cdot \nabla S=0$. This completes the proof of Theorem A.

In the noncompact case, we have the following criterion for the homogeneity of $M$.

Theorem 3.2. Assume that the Ricci eigenvalues $\rho_{1}, \rho_{2}, \rho_{3}$ are constant 
and distinct. Then $M$ is locally homogeneous if and only if the symmetric tensor $T=(S \cdot \nabla S) \otimes(S \cdot \nabla S)$ satisfies the conditions $\operatorname{tr} T=$ constant and $[S, T]=0$, where $[S, T]$ denotes the 2-form $\sum_{a}\left(S_{i}^{a} T_{a j}-T_{i}^{a} S_{a j}\right)$.

Proof. Note that the condition $[S, T]=0$ means that at least two of $\operatorname{div} X_{i}$ vanish, and recall the following fact. For any 3-dimensional nonunimodular Lie group $G$ with left invariant metric, if the Ricci eigenvalues are distinct, then two of $\operatorname{div} e_{i}$ 's of the eigenvector fields $e_{i}$ vanish ([M, p. 321]). Then the similar argument as before proves our theorem.

\section{§4. Proof of Theorem B}

We now discuss the degenerate case $\rho_{1}=\rho_{2} \neq \rho_{3}$. Theorem $B$ is an immediate consequence of the following assertion (If $\rho_{1}=\rho_{2}=\rho_{3}$, then Theorem B holds clearly, because in that case $M$ is a space of constant curvature).

THEOREM 4.1. Let $M$ be a 3-dimensional, connected, complete Riemann manifold. Assume that $\rho_{1}, \rho_{2}, \rho_{3}$ are constant, and now assume that $\rho_{1}=$ $\rho_{2} \neq \rho_{3}$. Define $\delta: M \rightarrow \mathbf{R}$ by the formula

$$
\delta=\rho_{3}-\frac{\|\nabla S\|^{2}}{2\left(\rho_{1}-\rho_{3}\right)^{2}},
$$

where $\|\nabla S\|^{2}=\sum_{a, b, c} S^{a b ; c} S_{a b ; c} . \quad$ Then $\delta \leq 0$, and the necessary and sufficient condition for $M$ to be locally homogeneous is that $\delta$ is constant. If $\delta=0$, then $\rho_{3} \geq 0$, and the isometry class of the universal covering manifold $\tilde{M}$ of $M$ is determined by $\rho_{1}, \rho_{3}$. If $\delta$ is negative constant, then $\rho_{1}=\rho_{2}=0$, the manifold $\tilde{M}$ is a Lie group with left invariant metric, and the isometry class of $\tilde{M}$ is determined by $\rho_{3}, \delta$.

Proof. Clearly, if $M$ is locally homogeneous, then $\delta$ is constant. To prove the converse, note that the function $\delta$ is nothing but the function defined in Lemma 1.2. Then by Proposition 2.1 we see that the constancy of $\delta$ ensures the local homogeneity of $M$. To prove the latter parts, suppose that $\delta=0$. Then Lemmas 2.2 and 2.4 imply that the isometry class of $\tilde{M}$ is determined by $\rho_{1}, \rho_{3}$. In the case $\delta \equiv$ negative constant, Lemmas 2.3 and 2.5 prove our assertion.

\section{§5. Global theory for the case $\rho_{1}=\rho_{2} \neq \rho_{3}$}

There are some cases in which only the constancy of $\rho_{1}, \rho_{2}, \rho_{3}$ ensures 
the local homogeneity of $M$. Indeed, we shall prove

Proposition 5.1. Let $M$ be a 3-dimensional, connected, compact Riemann manifold with constant Ricci eigenvalues $\rho_{1}, \rho_{2}, \rho_{3}$. If $\rho_{1}=\rho_{2}$, and if $\rho_{1} \geq 0$ or $\rho_{3} \leq 0$, then $M$ is locally homogeneous.

In order to prove this proposition we may assume that $\rho_{1}=\rho_{2} \neq \rho_{3}$. Then we have the function $\delta: M \rightarrow \mathbf{R}$ defined in Theorem 4.1, which coincides locally with the function $\delta$ in Lemma 1.2. Thus, to prove the local homogeneity of $M$ it suffices to show the constancy of $\delta$. This comes from

Lemma 5.2. Let $M$ be as in Proposition 5.1, and assume that $\rho_{1}=\rho_{2}$ $\neq \rho_{3}$.

(1) If $\rho_{3}<0$, then $\delta=$ negative constant, and $\rho_{1}=\rho_{2}=0$.

(2) If $\rho_{3}=0$, then $\delta \equiv 0$.

(3) If $\rho_{1}>0$, then $\delta \equiv 0$.

(4) If $\rho_{1}=0$, then $\delta=$ constant.

To prove Lemma 5.2 we prepare a lemma.

LEMma 5.3. Under the same hypothesis as in Lemma 5.2, if $\delta(x)<0$ for all $x \in M$, then we have $\rho_{1}=\rho_{2}=0$.

Proof. Let $X_{1}, X_{2}, X_{3}$ be the orthonormal vector fields on some finite covering manifold $\tilde{M}$ of $M$ such that $X_{3}$ is the eigenvector field belonging to $\rho_{3}$ and $X_{1}, X_{2}$ are locally as in Lemma 1.4. Then by the choice of $X_{1}, X_{2}$ the functions $d_{i j}^{k}=\left\langle\nabla_{X_{i}} X_{j}, X_{k}\right\rangle: \tilde{M} \rightarrow \mathbf{R}$ satisfy

$$
\begin{aligned}
& d_{33}^{1}=d_{33}^{2}=d_{11}^{3}=d_{22}^{3}=d_{31}^{2}=0, \quad \rho_{3}=2 d_{12}^{3} d_{23}^{1}, \\
& \delta=-\left(d_{12}^{3}-d_{23}^{1}\right)^{2}, \quad d_{12}^{3}>d_{23}^{1},
\end{aligned}
$$

and satisfy

$$
d_{12}^{3}+d_{23}^{1}>0 \text { at some point of } \tilde{M} \text { or } d_{12}^{3}+d_{23}^{1} \equiv 0 .
$$

Hence $(1,1)$ in Lemma 1.1 becomes $\rho_{1}=X_{1}\left(d_{22}^{1}\right)-\left(d_{22}^{1}\right)^{2}+X_{2}\left(d_{11}^{2}\right)-\left(d_{11}^{2}\right)^{2}$. By integrating this formula over $\tilde{M}$ we obtain $\rho_{1}=0$, because the right hand side can be written as $\operatorname{div}\left(d_{22}^{1} X_{1}+d_{11}^{2} X_{2}\right)$.

Proof of (1), Lemma 5.2. Assume $\rho_{3}<0$. From the expression for $\delta$ in the proof of Lemma 1.2 , we see that $\delta<0$ on $M$. Let $X_{1}, X_{2}, X_{3}, d_{i j}^{k}$ be as in the proof of Lemma 5.3. Then the functions $d_{i j}^{k}$ satisfy the same 
properties as before. We contend that $d_{12}^{3}, d_{23}^{1}$ are constant. This and Lemma 5.3 will prove (1). Now, since $\rho_{3}=2 d_{12}^{3} d_{23}^{1}$ and $X_{3}(\delta)=0$, we see that $d_{12}^{3}, d_{23}^{1}$ are $X_{3}$-invariant. Hence by $(3,1),(3,2)$ we see that the function $d_{22}^{1}$ satisfies the differential equation $\left(X_{3}\right)^{2}\left(d_{22}^{1}\right)+\left(\rho_{3} / 2\right) d_{22}^{1}=0$. Hence, for each integral curve $c(t)$ of $X_{3}$, the function $d_{22}^{1}(c(t))$ is of the form constant $e^{\sqrt{-\rho_{3} / 2} t}+$ constant $e^{-\sqrt{-\rho_{3} / 2} t}$. Therefore, since $M$ is assumed to be compact, the bounded function $d_{22}^{1}$ has to be identically zero. Similarly, $d_{11}^{2} \equiv 0$. Using $(1,3),(2,3)$ we conclude that $d_{12}^{3}, d_{23}^{1}$ are constant, as desired.

Proof of (2), Lemma 5.2. Assume that $\rho_{3}=0$, and assume on the contrary that $\delta \neq \equiv$. Now, consider the open submanifold $M_{0}=\{p \in M \mid \delta(p)$ $<0\}$ and the vector fields $X_{1}, X_{2}, X_{3}$ on some finite covering manifold $\tilde{M}_{0}$ of $M_{0}$ as in the proof of Lemma 5.3. Then we have the functions $d_{i j}^{k}=$ $\left\langle\nabla_{X_{i}} X_{j}, X_{k}\right\rangle: \tilde{M}_{0} \rightarrow \mathbf{R}$ satisfying the same properties as before. By the assumption $\rho_{3}=0$, using the latter part of the properties in Lemma 1.4, we get $d_{12}^{3}>0$ and $d_{23}^{1}=0$. Then $(2,3)$ of Lemma 1.1 yields $d_{22}^{1}=0$, and hence by $(1,1)$ we get $\rho_{1}=X_{2}\left(d_{11}^{2}\right)-\left(d_{11}^{2}\right)^{2}$. Furthermore, by $(1,3)$ we have $X_{2}\left(d_{12}^{3}\right)-d_{11}^{2} d_{12}^{3}=0$. We contend that $\rho_{1}<0$. In fact, let $p$ be the point where $\delta$ takes its minimum. Then $d_{12}^{3}$ is maximal at $p$, and hence $X_{2}\left(d_{12}^{3}\right)(p)$ $=0$, and $\left(X_{2}\right)^{2}\left(d_{12}^{3}\right)(p) \leq 0$. Therefore $d_{11}^{2}(p)=0, X_{2}\left(d_{11}^{2}\right)(p) \leq 0$, and hence $\rho_{1} \leq 0$. Recalling the assumption $\rho_{1} \neq \rho_{3}$, we get $\rho_{1}<0$. Thus we can consider the non-empty open set $V=\left\{p \in \tilde{M}_{0} \mid\left(d_{11}^{2}\right)^{2}(p)<-\rho_{1}\right\}$. We shall show that the volume of $V$ is infinite. This contradiction will prove our assertion (2). To estimate the volume of $V$, we denote by $p(t)$ the integral curve for $X_{2}$ through a point $p$. We contend that for any point $p \in V$, the curve $p(t)$ is defined for all $t \in \mathbf{R}$, and that $p(t) \in V$ for all $t$. In fact, from the differential equation $\rho_{1}=X_{2}\left(d_{11}^{2}\right)-\left(d_{11}^{2}\right)^{2}$ and the initial condition $\left|d_{11}^{2}(p)\right|<\sqrt{-\rho_{1}}$, we observe that $\left|d_{11}^{2}(p(t))\right|<\sqrt{-\rho_{1}}$ as far as $p(t)$ is defined. Hence, from the differential equation $X_{2}\left(d_{12}^{3}\right)-d_{11}^{2} d_{12}^{3}=0$ we see that the function $d_{12}^{3}(p(t))=\sqrt{-\delta(p(t))}$ does not accumulate to zero in finite $t$. Consequently, the curve $p(t)$ is defined for all $t$ and lies in $V$. The above argument also shows that $X_{2}$ is transversal to the 2-dimensional submanifold $V_{0}=\left\{p \in \tilde{M}_{0} \mid d_{11}^{2}(p)=0\right\}$, and each curve $p(t)$ in $V$ intersects with $V_{0}$ once and only once. Furthermore, for any curve $p(t)$ starting at $p \in V_{0}$, we have $\left(\operatorname{div} X_{2}\right)(p(t))=-d_{11}^{2}(p(t))>0$ for any $t>0$. Thus we have to conclude that the volume of $V$ is infinite, as desired. Assertion (2) is proved. 
Proof of (3) and (4), Lemma 5.2. Assume that $\rho_{1} \geq 0$. By (1), (2), in order to prove (3), (4), we may assume that $\rho_{3}>0$. Now, suppose that $\delta \not \equiv 0$. Then we can consider the submanifold $M_{-}=\{p \in M \mid \delta(p) \leq-\varepsilon\}$ with some small $\varepsilon>0$ and the vector fields $X_{1}, X_{2}, X_{3}$ on some finite covering manifolds $\tilde{M}_{-}$as in the proof of Lemma 5.3. Noting that the functions $d_{i j}^{k}=\left\langle\nabla_{X_{i}} X_{j}, X_{k}\right\rangle: \tilde{M}_{-} \rightarrow \mathbf{R}$ satisfy the same properties as in the proof of Lemma 5.3, we see that the assumption $\rho_{3}>0$ yields $d_{12}^{3}, d_{23}^{1}>0$, and from $(2,3),(1,3)$ of Lemma 1.1 we have $X_{1}\left(d_{23}^{1}\right)=-d_{22}^{1}\left(d_{12}^{3}-d_{23}^{1}\right)$, $X_{2}\left(d_{12}^{3}\right)=d_{11}^{2}\left(d_{12}^{3}-d_{23}^{1}\right)$. We contend that the inner product of the vector field $d_{22}^{1} X_{1}+d_{11}^{2} X_{2}$ and the vector field $\operatorname{grad} \delta$ is given by

$$
\left\langle d_{22}^{1} X_{1}+d_{11}^{2} X_{2}, \operatorname{grad} \delta\right\rangle=4\left(d_{12}^{3}+d_{23}^{1}\right)\left(\frac{d_{12}^{3}\left(d_{22}^{1}\right)^{2}+d_{23}^{1}\left(d_{11}^{2}\right)^{2}}{\rho_{3}}\right) \delta,
$$

which is clearly nonpositive at every point, and which is zero if and only if $d_{22}^{1}=d_{11}^{2}=0$. In fact, applying $X_{1}, X_{2}$ to $\rho_{3}=2 d_{12}^{3} d_{23}^{1}$, we have $X_{1}\left(d_{12}^{3}\right)$ $=\left(d_{12}^{3} / d_{23}^{1}\right) d_{22}^{1}\left(d_{12}^{3}-d_{23}^{1}\right), X_{2}\left(d_{23}^{1}\right)=-\left(d_{23}^{1} / d_{12}^{3}\right) d_{11}^{2}\left(d_{12}^{3}-d_{23}^{1}\right)$. Then our contention is easily verified. Now we can prove assertion (3). Since $\rho_{1}=$ $\operatorname{div}\left(d_{22}^{1} X_{1}+d_{11}^{2} X_{2}\right)$, integrating $\rho_{1}$ over $\tilde{M}_{-}$and using the integral formula, we get

$$
\rho_{1} \operatorname{vol}\left(\tilde{M}_{-}\right)=\int_{\partial \tilde{M}_{-}}\left\langle d_{22}^{1} X_{1}+d_{11}^{2} X_{2}, \operatorname{grad} \delta\right\rangle \frac{1}{\|\operatorname{grad} \delta\|}
$$

Since the integrand of the right hand side is nonpositive as proved above, we obtain $\rho_{1} \leq 0$. This contradiction proves (3). In order to prove (4), it suffices to verify that $d_{12}^{3}, d_{23}^{1}$ are constant and hence that $d_{22}^{1}=d_{11}^{2}=0$. This also follows from the above integral expression. Indeed, if $\rho_{1}=0$, then the integrand has to be zero identically on the boundary, and hence $d_{22}^{1}=d_{11}^{2}=0$. This proves (4) and completes the proof of Lemma 5.2.

Lemma 5.2 , (1) has the following corollary:

Proposition 5.4. Let $M$ be a compact Riemann manifold with constant Ricci eigenvalues $\rho_{1}, \rho_{2}, \rho_{3}$. If $\rho_{1}=\rho_{2} \neq \rho_{3}$ and $\rho_{3}<0$, then $\rho_{1}=\rho_{2}=0$.

Remark. Proposition 5.1 still holds for non-compact but comlete $M$ with $\rho_{1}=\rho_{2}>0, \rho_{3}=0$. This fact is essentially proved in [Se]. In our notation in the proof of Lemma 5.2, (2), this is verified as follows: It suffices to prove $\delta \equiv 0$. Let $p \in M_{0}$, if any. By $(1,1)$, (1,3) we observe that the function $d_{12}^{3}(p(t))$ is of the form 


$$
d_{12}^{3}(p(t))=\frac{\text { const }}{\cos \left(\sqrt{\rho_{1}} t+\text { const }\right)},
$$

where $p(t)$ is the integral curve for $X_{2}$ through $p$. This expression shows, on the one hand, that the curve $p(t)$ does not approach the boundary of $\tilde{M}_{0}$, and hence that $p(t)$ is defined for all $t$, and on the other hand, that the function $d_{12}^{3}(p(t))$ is not defined for some $t$. This contradicts to the fact that $d_{12}^{3}$ is defined on the whole $\tilde{M}_{0}$. Consequently $M_{0}=\emptyset$, i.e. $\delta \equiv 0$, as desired.

Note that Proposition 5.1 does not hold generally in the noncompact case. In fact, there exist examples of non-homogeneous, complete metric on $\mathbf{R}^{3}$ with $\rho_{1}=\rho_{2}=-1, \rho_{3}=0$ ([Se], also [T]). K. Sekigawa found these examples in his study of Nomizu conjecture in dimension 3. Moreover, the recent result by $O$. Kowalski, F. Tricerri and L. Vanhecke ([KTV]) asserts that the set $\mathscr{M}\left(\mathbf{R}^{3} ;-1,-1,0\right)$ of isometry classes of complete metrics on $\mathbf{R}^{3}$ with Ricci eigenvalues $\rho_{1}=\rho_{2}=-1, \rho_{3}=0$ is infinite dimensional. On the other hand, Proposition 5.1 (with Theorem B) implies that the set $\mathscr{M}\left(S^{3} ; \rho_{1}, \rho_{2}, 1\right), 0<\rho_{1}=\rho_{2} \neq 1$, for example, is just one point (the isometry class of some left invariant metric on $S U(2)$ ). We will also see that the set $\mathscr{M}\left(M ; \rho_{1}, \rho_{2},-1\right)$ for compact $M$ is empty if $0 \neq \rho_{1}=\rho_{2}$ $\neq-1$ (Proposition 5.4). Much yet remains to be studied about the set $\mathscr{M}\left(M ; \rho_{1}, \rho_{2}, \rho_{3}\right)$.

\section{§6. Some curvature homogeneous metrics on $\mathbf{R}^{3}$}

We give 3-dimensional, complete Riemann manifolds (diffeomorphic to $\mathbf{R}^{3}$ ) which are not homogeneous, but have distinct, constant Ricci eigenvalues. To give such examples, let $\rho_{1}, \rho_{2}, \rho_{3}$ be three distinct real numbers such that the numbers

$$
A=\frac{\rho_{1}+\rho_{2}-\rho_{3}}{2}, \quad B=\frac{\rho_{1}-\rho_{3}}{\rho_{3}-\rho_{2}}, \quad C=-\frac{\left(\rho_{1}+\rho_{2}\right)\left(\rho_{3}-\rho_{2}\right)^{2}}{\left(\rho_{2}-\rho_{1}\right)^{2}} .
$$

satisfy the inequalities $A>0, C>0, A+B C>0$. Then there exists a complete, non-homogeneous Riemann metric $g$ on $\mathbf{R}^{3}$ with constant Ricci eigenvalues $\rho_{1}, \rho_{2}, \rho_{3}$. In fact, consider the vector fields $X_{1}, X_{2}, X_{3}$ on $\mathbf{R}^{3}$ defined by

$$
X_{1}=\frac{\partial}{\partial x}, \quad X_{2}=\frac{\partial}{\partial y},
$$




$$
X_{3}=\frac{\partial}{\partial z}+(x \phi(z)+y f(z)) \frac{\partial}{\partial x}+(x g(z)+B y \phi(z)) \frac{\partial}{\partial y},
$$

where $\phi(z)$ is a solution of the differential equation

$$
\frac{d \phi}{d z}+(1+B)\left(\phi^{2}-C\right)=0 \text { satisfying } \phi^{2}<C,
$$

and $f, g$ are the functions chosen so that

$$
f^{2}-g^{2}=2\left(\rho_{1}+(1+B) C\right), \quad(f+g)^{2}=4\left(A+B \phi^{2}\right), f+g>0 .
$$

Specifically, if $\rho_{1}=-5 / 6, \rho_{2}=7 / 12, \rho_{3}=-9 / 4$, then

$\phi=\frac{1-e^{-z}}{1+e^{-z}}, \quad f=\sqrt{1-\frac{1}{2} \phi^{2}}-\frac{1}{6 \sqrt{1-\frac{1}{2} \phi^{2}}}, \quad g=\sqrt{1-\frac{1}{2} \phi^{2}}+\frac{1}{6 \sqrt{1-\frac{1}{2} \phi^{2}}}$.

Let $\mathbf{g}$ be the Riemann metric on $\mathbf{R}^{3}$ such that $X_{1}, X_{2}, X_{3}$ are orthonormal. Then the Riemann manifold $\left(\mathbf{R}^{3}, \mathbf{g}\right)$ is complete and non-homogeneous, but has constant Ricci eigenvalues $\rho_{1}, \rho_{2}, \rho_{3}$. The completeness follows from the fact that there exist constants $K, L$ such that

$$
u^{2}+v^{2}+w^{2} \leq K\left(x^{2}+y^{2}+z^{2}\right)+L
$$

for any tangent vector $Y=u \partial / \partial x+v \partial / \partial y+w \partial / \partial z$ at $(x, y, z)$ satisfying $\boldsymbol{g}(Y, Y)=1$. Since $\operatorname{div} X_{3}=(1+B) \phi$ is nonconstant, the non-homogeneity is obvious.

Finally, we should mention the following

Problem. Give a compact connected Riemann manifold of dimension 3 which is not locally homogeneous, but has constant Ricci eigenvalues.

This seems to be difficult, but is interesting as a problem of global analysis on manifolds.

\section{REFERENCES}

[KTV] O. Kowalski, F. Tricerri and L. Vanhecke, Curvature homogeneous Riemannian manifolds, preprint.

[M] J. Milnor, Curvatures of left invariant metrics on Lie groups, Advances in Math., 21 (1976), 293-329.

[O] B. O'Neill, The fundamental equations of a submersion, Michigan Math. J., 13 (1966), 459-469.

[Se] K. Sekigawa, On some 3-dimensional Riemannian manifolds, Hokkaido Math. J., 2 (1973), 259-270.

[Si] I. M. Singer, Infinitesimally homogeneous spaces, Comm. Pure Appl. Math., 13 (1960), 685-697. 
[T] H. Takagi, On curvature homogeneity of Riemannian manifolds, Tôhoku Math. J., 26 (1974), 581-585.

[TV] F. Tricerri and L. Vanhecke, Curvature homogeneous Riemannian manifolds, Ann. Sci. École Norm. Sup., 22 (1989), 535-554.

Department of Mathematics

College of General Education

Nagoya University

Chikusa-ku, Nagoya 464-01

Japan 